\title{
Chronic hyponatremia based on maxillary sinus mass
}

\author{
Darija Tudor ${ }^{1}$, Iva Kolombo², Ana Tot ${ }^{1}$, Drasko Cikojevic ${ }^{3}$, Marko Simunovic ${ }^{\mathbb{D} 1,4}$ and \\ Veselin Skrabic ${ }^{1,4}$ \\ 1Department of Pediatrics, University Hospital of Split, Split, Croatia, ${ }^{2}$ Department of Pediatrics, Šibenik General \\ Hospital, Šibenik, Croatia, ${ }^{3}$ Department of Otorhinolaryngology, University Hospital of Split, Split, Croatia, and \\ ${ }^{4}$ Department of Pediatrics, University of Split School of Medicine, Split, Croatia
}

Correspondence should be addressed to $V$ Skrabic

Email

vskrabic@kbsplit.hr

\section{Summary}

This is a case report of a child with chronic hyponatremia due to the syndrome of inappropriate antidiuretic hormone secretion (SIADH) as a paraneoplastic manifestation of olfactory neuroblastoma (OFN). We hereby report a clinical presentation as well as a pragmatic approach to one of the most common electrolytic disorders in the pediatric population and have emphasized the necessity of involving the sinonasal area in the diagnostic procedure while evaluating possible causes of SIADH. This report indicates that the chronicity of the process along with the gradual onset of hyponatremia occurrence is responsible for the lack of neurological symptoms at the moment of disease presentation.

\section{Learning points:}

- Hyponatremia is not infrequently attributed to SIADH.

- Paraneoplastic syndromes are uncommon but they should be considered in the differential diagnosis of pediatric SIADH.

- Chronic insidious hyponatremia may not be associated with clear neurological symptoms despite its severity.

\section{Background}

Hyponatremia, defined by sodium levels of $<135 \mathrm{mmol} / \mathrm{L}$, is one of the most common electrolytic disorders seen in clinical practice. Hypoosmolality, caused by decreased serum sodium levels, results in cerebral edema and possible cerebral herniation (1). Among different causes of hyponatremia in the pediatric population, the syndrome of inappropriate antidiuretic hormone secretion (SIADH) is reported to be one of the most common (2). SIADH refers to states of hypoosmolar hyponatremia caused by nonphysiological stimuli for arginine vasopressin (AVP) secretion. It implies euvolemic states characterized by impairedfreewaterexcretionalongwithlessthan maximally diluted urine and natriuresis in the absence of heart, renal, or endocrine dysfunction (3). The first case of SIADH was reported in 1957 by Schwartz and Bartter in two adult patients with bronchogenic carcinoma. In the pediatric population, SIADH is most often the result of pulmonary disorders like pneumonia, but also CNS disorders including pituitary tumors or following transsphenoidal surgery, malignancies, and medications (3). While hyponatremia may be the most common electrolyte abnormality seen in children, it should be acknowledged that it is much rarer in children than in adults. Therefore, every pediatric case of unexplained hyponatremia should be evaluated for potential tumor-related SIADH (2). In children affected by SIADH, after a primary pulmonary lesion has been excluded, a further diagnostic procedure must include the study of the sinonasal area. A tumor in the mentioned 
region - olfactory neuroblastoma, can present with paraneoplastic syndrome-mediated SIADH (4). Olfactory neuroblastoma, also known as esthesioneuroblastoma, is a rare malignancy of neuroectodermal origin with low frequency in the pediatric population (5). The tumor arises from the specialized sensory neuroepithelial cells of the olfactory membrane that are normally found in the upper part of the nasal cavity (6). It has been estimated that SIADH appears in only 26 of the 1300 reported cases of olfactory neuroblastoma mentioned in the literature, which implies a rather small proportion of only $2 \%$. In approximately half of the cases, SIADH had been presented before the diagnosis of the tumor was established (7). After an extensive review of the literature, we would like to point out that there are only a few published cases of SIADH caused by olfactory neuroblastoma in the pediatric population, amongst which our patient was the youngest. She was also the only one without any neurological symptoms (6). The aim of this case report is to show a very unusual presentation of the most common electrolytic disorder caused by an extremely rare condition in children.

\section{Case presentation}

We report a case of an 11.1-year-old patient, female, a child of healthy parents, normally developed with no significant premorbid history. She was presented at our Pediatrics Emergency Department with recurring epistaxis, a fourth episode in a month and a half period. She also reported a mild generalized pain especially in the abdomen with the stool of softer consistency during the mentioned period. Afterward, we learned that she had cravings for salty food for a longer time. Clinical status at the moment of admission was age: 11.1 years, body height: $151 \mathrm{~cm} \mathrm{(+0.87}$ S.D.), body weight: $37 \mathrm{~kg}$, BMI: $16.2 \mathrm{~kg} / \mathrm{m}^{2}$ (-0.58 S.D.), blood pressure: 120/80 $\mathrm{mmHg}$, oxygen saturation: $99 \%$, heart rate: $134 / \mathrm{min}$, temperature: $36.9^{\circ} \mathrm{C}$, respiratory rate: 20/min, recapillarization time: < 2 s. She was in good general condition with unremarkable heart, pulmonary, and abdominal physical status.

\section{Investigation}

Routine blood investigations revealed hyponatremia (128 mmol/L), hypochloremia (94 mmol/L), serum hypoosmolality ( $258 \mathrm{mOsm} / \mathrm{kg}$ ) with high urine osmolality (902 mOsm/kg), and significant natriuresis $(234 \mathrm{mmol} / \mathrm{L})$. Values of other remaining electrolytes, glucose, acid-basic status as well as hepatic and renal function parameters were within referent ranges. Chest X-ray showed no pathological substrate. Further laboratory analysis excluded thyroid or adrenal dysfunction (short adrenocorticotropin test, aldosterone, and plasma renin activity were all within referent values). Neuroradiological imaging - both CT and MRI detected a tumor formation in the right maxillary sinus (Figs 1 and 2).

\section{Treatment}

Initial treatment approach included parenteral sodium replacement with hypertonic saline which initially led to a further decline in sodium values (111 mmol/L). It was only fluid restriction accompanied by administration of high doses of sodium (up to $12.6 \mathrm{mmol} / \mathrm{kg} /$ day) that accomplished a mild rise in sodium levels (up to $115 \mathrm{mmol} / \mathrm{L}$ ) but still with excessive natriuresis (215 mmol/L). Maxillary antrostomy was performed, and most of the tumor mass was removed which led to prompt normalization of serum sodium levels (135 $\mathrm{mmol} / \mathrm{L})$ and cessation of natriuresis (Figs 3 and 4).

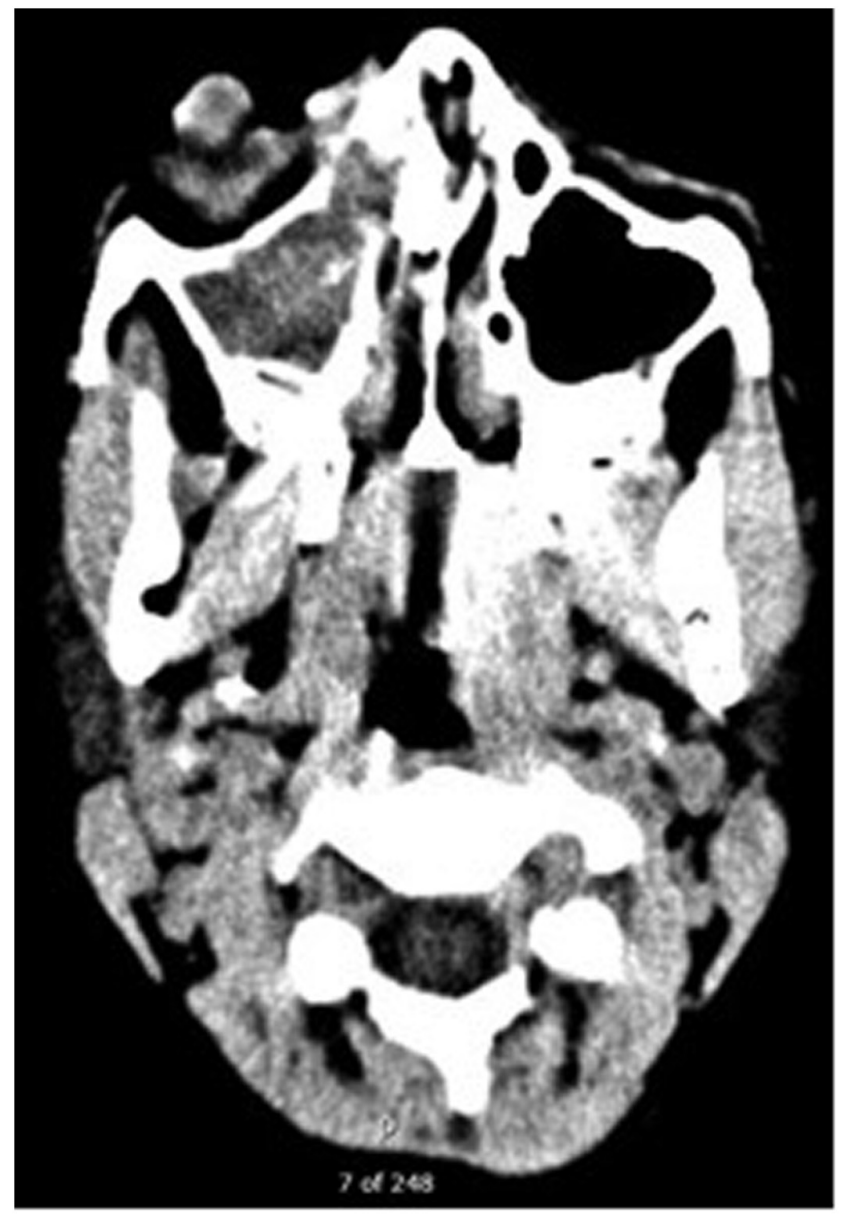

Figure 1

CT image of neuroblastoma in right maxillary sinus. 


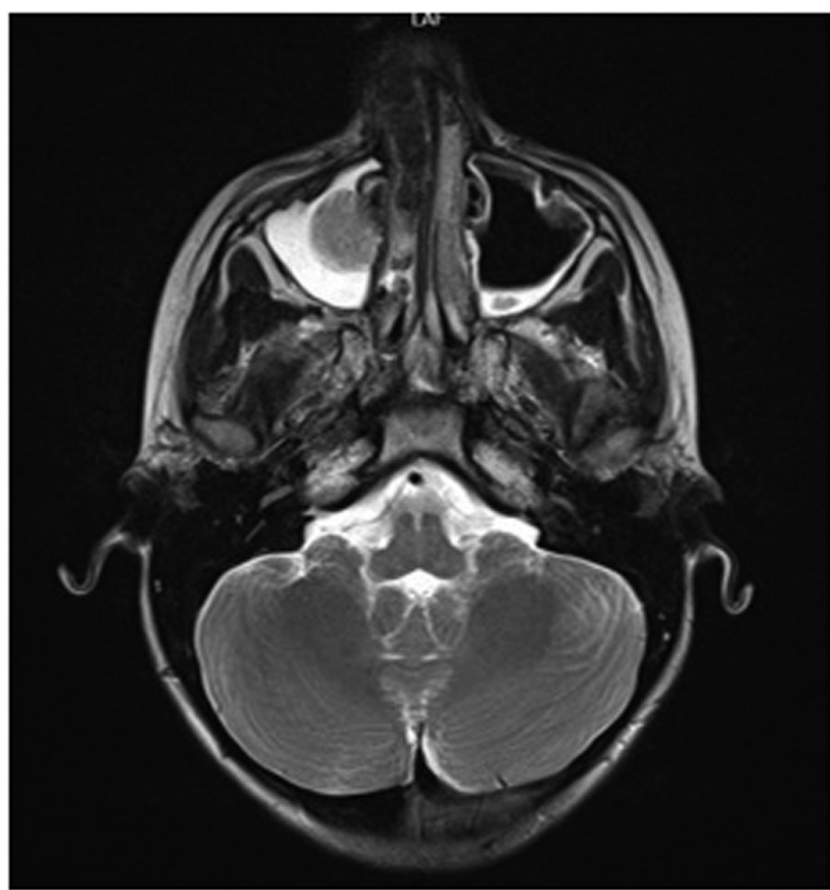

Figure 2

MR image of neuroblastoma in right maxillary sinus.

\section{Outcome and follow-up}

Pathohistological analysis established the diagnosis of olfactory neuroblastoma, grade I according to Hyams classification, MYCN negative, without chromosome 1 deletion (4). Given the presence of $4 \mathrm{~mm}$ nodular lesions in the lungs detected by CT, treatment according to NB 2004 protocol with N5/N6 cycles was started and four cycles of chemotherapy were to be performed.

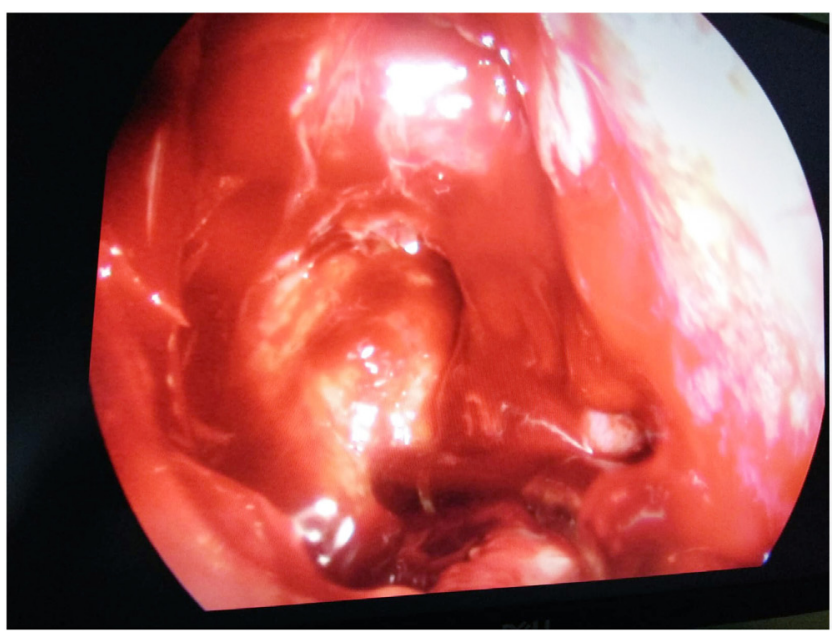

Figure 3

Intraoperative image of affected maxillary sinus.

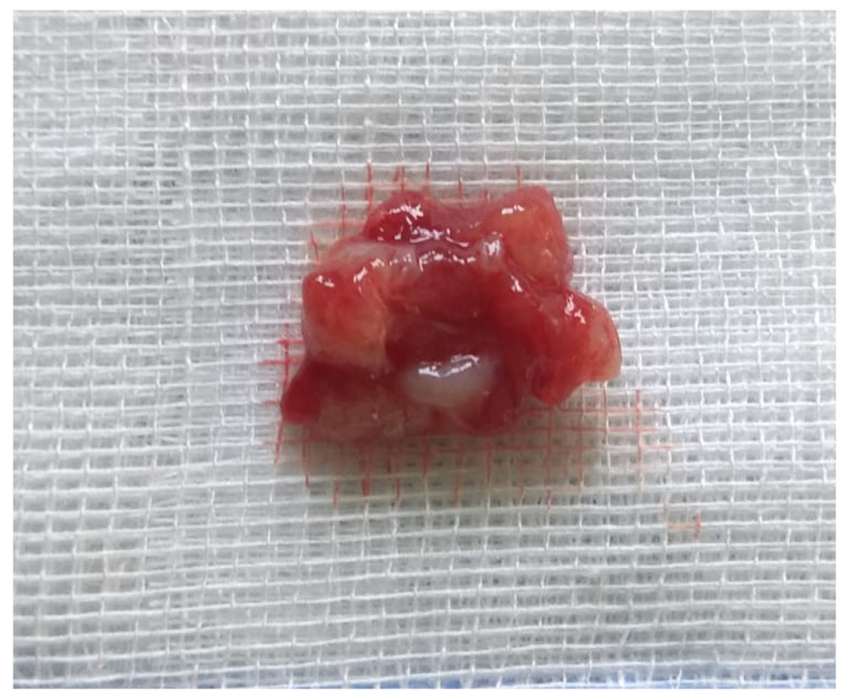

Figure 4

Extracted tumor mass.

\section{Discussion}

Although SIADH stands out among the most common causes of hyponatremia in the pediatric population, it should be borne in mind that its diagnosis is made per exclusionem (2). Initial approach to hyponatremia requires the exclusion of pseudohyponatremia resulting from severe hyperproteinemia, hyperlipidemia, hyperglycemia, or administration of mannitol or radiocontrast agents. If hyponatremia is associated with serum hypoosmolality, the next step is to measure the osmolality of urine in order to determine whether the ability of free water excretion is impaired (3). Also, diseases that cause reduced effective circulating volume, renal impairment, endocrine disorders such as hypothyroidism or adrenal insufficiency should be ruled out. The most common laboratory finding associated with primary adrenal insufficiency is hyponatremia (90\% of cases) along with hyperkalemia (50\% of cases) and hypoglycemia (30\% of cases). Given the fact that the diagnosis of adrenal insufficiency cannot be ruled out by the absence of hyperkalemia and hypoglycemia, initial presentation with hyponatremia accompanied by high natriuresis may imitate SIADH (8). Restriction of fluid intake as the first line of therapy for SIADH may, in patients with adrenal insufficiency, precipitate a lifethreatening condition, which emphasizes the importance of correct diagnosis of these clinical entities (8). A further challenge in establishing the diagnosis is to distinguish SIADH from cerebral/renal salt loss syndrome (C/RSW) due to a different therapeutic approach. CSW is a less common condition that most often appears as a symptom 
of CNS disease. It is characterized by primary natriuresis and hypovolemia as a result of inappropriate and excessive secretion of natriuretic peptides (1). However, due to the activation of compensatory mechanisms (reninangiotensin-aldosterone system and increased vasopressin production), signs of volume depletion are not always present, and there is no biochemical parameter that would reliably distinguish CSW and SIADH at the time of disease presentation (1). However, the lack of therapeutic response to saline infusions seen in our patient, accompanied by the absence of even mild hypovolemia as well as a positive response to fluid restriction, altogether negates the possibility of CSW in this concrete case.

Differential diagnosis of SIADH requires a detailed and pragmatic approach given the variety of clinical conditions that can lead to it (3). Pediatric SIADH is most often the result of pulmonary diseases (pneumonia, bronchiolitis, asthma, positive pressure ventilation), CNS infections, and head trauma (2). Malignancies can also cause SIADH in a pediatric population. SIADH, as a paraneoplastic syndrome, is most commonly associated with small cell lung cancer but, once the primary pulmonary lesion has been ruled out, the sinonasal region should be included in the diagnostic procedure (6). A malignant neuroectodermal tumor originating from the cells of the roof of the nasal cavity - olfactory neuroblastoma, may, although rarely, be associated with paraneoplastic syndromes. It is currently known to cause SIADH, ectopic ACTH syndrome, hypercalcemia due to parathyroid hormonerelated peptide (PTHrP) secretion, and hypertension due to catecholamine secretion (6). Although it can occur at any age, its incidence peaks in the second and sixth decades of life without specific gender predilection. Common clinical presentation includes nasal obstruction, epistaxis, sinusitis, and headache - relatively nonspecific clinical symptoms considered responsible for often late diagnosis (4).

It is important to point out that very high urine osmolality, such as our patient's, suggests ectopic AVP section, which has been proven in several previous cases of ethesioneuroblastoma $(9,10,11)$.

The primary symptoms of hyponatremia are nonspecific as well and, therefore, can easily be overlooked - headache, nausea, vomitus, generalized weakness. Symptom progression includes convulsions, respiratory arrest, noncardiogenic pulmonary edema, and decortication (12). The most serious complication of hyponatremia is hyponatremic encephalopathy, and its primary symptoms are due to cerebral edema (3). The average value of serum sodium in children with hyponatremic encephalopathy is $120 \mathrm{mmol} / \mathrm{L}$, significantly higher than seen in adults: $111 \mathrm{mmol} / \mathrm{L}$ (12). Since our patient did not have a neurological status disorder or any of the above symptoms during hyponatremia with $128 \mathrm{mmol} / \mathrm{L}$ values, it was concluded that her condition was a chronic one. Subsequently, anamnestic data on several months of craving for salty foods were obtained, though this symptom is unusual and is not a typical manifestation of hyponatremia due to SIADH (3).

Treatment of hyponatremia should be carried out with caution against hasty correction of serum sodium value with the aim of preventing central pontine myelinolysis (3). In order to prevent that adverse effect, it is recommended that the degree of increase in sodium values does not exceed $10-12 \mathrm{mmol} / \mathrm{L}$ in a $24-\mathrm{h}$ period in patients with a normal risk of osmotic demyelination (13).

Although the basis of SIADH treatment is the fluid restriction with increased salt intake per os, primary treatment involves the treatment of the underlying condition, which in this case involves surgical removal of the tumor with adjuvant radiotherapy and/or chemotherapy depending on the stage of the disease $(4,13)$. After an extensive review of the literature, it is important to point out that the combination of high urine osmolality and high urine sodium seen in our patient indicates a very low renal electrolyte-free water excretion with a subsequent very high urine: plasma electrolyte ratio which is a predictor of failure to respond to fluid restriction. Therefore, more effective treatment of this patient's hyponatremia would have been the use of aquaretic agents (e.g. vaptans or urea) (13).

\section{Declaration of interest}

The authors declare that there is no conflict of interest that could be perceived as prejudicing the impartiality of this case report.

\section{Funding}

This work did not receive any specific grant from any funding agency in the public, commercial or not-for-profit sector.

\section{Patient consent}

A written informed consent has been obtained from the patient's guardian for publication of this article and accompanying images.

\section{Author contribution statement}

D T, I K and A S wrote the manuscript. D C, M S and $V \mathrm{~S}$ revised the manuscript. I K, D C, M S and V S were involved in the care of the patient. 


\section{References}

1 Bussmann C, Bast T \& Rating D. Hyponatraemia in children with acute CNS disease: SIADH or cerebral salt wasting? Child's Nervous System 200117 58-62; discussion 63. (https://doi.org/10.1007/ s003810100478)

2 Wattad A, Chiang ML \& Hill LL. Hyponatremia in hospitalized children. Clinical Pediatrics 199231 153-157. (https://doi. org/10.1177/000992289203100305)

3 Moritz ML. Syndrome of Innapropriate antidiuresis. Pediatric Clinics of North America 201966 209-226. (https://doi.org/10.1016/j. pcl.2018.09.005)

4 Yumusakhuylu AC, Binnetoglu A, Topuz MF, Bozkurtlar EB, Baglam T $\&$ Sari M. Syndrome of inappropriate antidiuretic hormone secretion associated with olfactory neuroblastoma. Journal of Craniofacial Surgery 201324 2189-2193. (https://doi.org/10.1097/ SCS.0b013e3182a41c52)

5 Benoit MM, Bhattacharyya N, Faquin W \& Cunningham M. Cancer of the nasal cavity in the pediatric population. Pediatrics 2008121 e141-e145. (https://doi.org/10.1542/peds.2007-1319)

6 Kunc M, Gabrych A, Czapiewski P \& Sworczak K. Paraneoplastic syndromes in olfactory neuroblastoma. Contemporary Oncology 2015 19 6-16. (https://doi.org/10.5114/wo.2015.46283)

7 Gabbay U, Leider-Trejo L, Marshak G, Gabbay M \& Fliss DM. A case and a series od published cases of esthesioneuroblastoma (ENB) in which long-standing paraneoplastic SIADH had preceded ENB diagnosis. Ear, Nose and Throat Journal 201392 10-12. (https://doi. org/10.1177/014556131309200103)

8 Pintaldi S, Lora A, Vecchiato K, Taddio A \& Barbi E. SIADH versus adrenal insufficiency: a life-threatening misdiagnosis. Italian Journal of Pediatrics 201945 23. (https://doi.org/10.1186/s13052-019-0614-1)

9 Nakano T, Motoshita J, Sawada F, Okabe M, Tamae A, Hiramatsu S, Kodama S, Shiratsuchi H, Yasumatsu R, Nakashima T, et al. Syndrome of inappropriate antidiuretic hormone secretion in a case of olfactory neuroblastoma without anti-diuretic hormone immunoreactivity: a case report and review of the literature. Auris, Nasus, Larynx $2017 \mathbf{4 4}$ 771-774. (https://doi.org/10.1016/j.anl.2016.11.012)

10 Senchak A, Freeman J, Ruhl D, Senchak J \& Klem C. Low-grade esthesioneuroblastoma presenting as SIADH: a review of atypical manifestations. Otolaryngology Case Reports 20122012 1-6.

11 Hoorn EJ, Monserez DA, Fenton RA, Overdevest I, Apperloo AJ, Zietse R \& Hardillo JA. Olfactory neuroblastoma with hyponatremia. Journal of Clinical Oncology 201533 e88-e92. (https://doi.org/10.1200/ JCO.2013.49.1464)

12 Moritz ML \& Ayus JC. New aspects in the pathogenesis, prevention, and treatment of hyponatremic encephalopathy in children. Pediatric Nephrology 201025 1225-1238. (https://doi.org/10.1007/s00467-0091323-6)

13 Verbalis JG, Goldsmith SR, Greenberg A, Korzelius C, Schrier RW, Sterns RH \& Thompson CJ. Diagnosis, evaluation, and treatment of hyponatremia: expert panel recommendations. American Journal of Medicine 2013126 (Supplement 1) S1-42. (https://doi.org/10.1016/j. amjmed.2013.07.006)

Received in final form 2 July 2021

Accepted 13 July 2021 